\title{
Monocular-contingent and binocular-contingent aftereffects
}

\author{
S. L. JIAO, C. HAN, Q. C. JING, and R. OVER \\ Institute of Psychology, Chinese Academy of Sciences, Beijing, People's Republic of China
}

\begin{abstract}
Alternate monocular and binocular exposure to complementary stimulation can yield opposite but coexisting aftereffects that are contingent on whether the test display is viewed with one eye or two eyes. The motion aftereffect was studied by adapting each eye separately to a contracting spiral and both eyes together to an expanding spiral. The stationary test spiral subsequently appeared to be expanding when viewed monocularly, but to be contracting when it was seen with both eyes open. With respect to the McCollough effect, after monocular exposure to red-vertical and green-horizontal gratings and binocular exposure to red-horizontal and green-vertical gratings, the appearance of the color of the test gratings when viewed with one eye was different from that when viewed with both eyes. Opposite, coexisting aftereffects induced by complementary stimulation can be interpreted as evidence that there are unique binocular aspects to visual function.
\end{abstract}

Several psychophysical paradigms have been used in attempts to separate monocular and binocular aspects of information processing within the visual system. For example, the pattern and depth effects obtained when viewing random-dot stereograms can be attributed to mechanisms that integrate inputs from the two eyes, since neither figure nor depth is visible within the display presented to either eye (Julesz, 1971). Interocular transfer of spatial aftereffects has also been interpreted by reference to binocular function. Stationary lines appear to move upwards following exposure to downward moving lines when the two displays are viewed either with a single eye or with both eyes. This aftereffect can also be obtained with dichoptic stimulation when one eye views the moving pattern and the other eye views the stationary display. It has been claimed that the neural substrate for transfer of the aftereffect lies at or beyond the site in the visual system where inputs from the two eyes first converge, since transfer could occur only to the extent that cells adapted by stimulation of one eye mediate perception of the display later shown to the other eye. The extent to which interocular transfer is partial rather than complete has been taken as an index of the contribution of monocular as opposed to binocular detectors.

Long (1979) questioned the claim that binocularity in visual function could be identified through mea-

\footnotetext{
C. Han is affiliated with the Department of Psychology, Beijing University. R. Over was on study leave from La Trobe University, Australia, and his participation in the project was made possible by support from the Institute of Psychology, Chinese Academy of Sciences. The authors are grateful to Beijing University for the use of laboratory facilities. Requests for reprints should be sent to Q. C. Jing (C. C. Ching), Institute of Psychology, Chinese Academy of Sciences, Beijing, People's Republic of China.
}

surement of interocular transfer of aftereffects. He argued that phenomenal overlap of the two monocular fields might cause the observer to attribute continuing input from the closed but still signaling eye to the open eye. However, this objection can be ruled out, because interocular transfer of an aftereffect occurs when the continued input from the previously adapted eye has been eliminated through pressure blinding (Barlow \& Brindley, 1963; Blake \& Fox, 1972). Further evidence that binocular functioning is necessary for interocular transfer has come from demonstrations that stereoblind observers, who can be presumed from independent evidence to lack detectors able to integrate inputs from the two eyes (e.g., Lema \& Blake, 1977), show little or no interocular transfer of aftereffects (Movshon, Chambers, \& Blakemore, 1972; Mitchell \& Ware, 1974).

Other investigators have sought to identify separate components within visual processing by comparing aftereffects under four inspection-test conditions: monocular-monocular (M-M); monocular-binocular (M-B); binocular-binocular (B-B); and binocularmonocular (B-M). Wolfe and Held (1981) concluded that there existed purely binocular detectors in addition to the monocular and binocular detectors that can be excited through stimulation of either eye or both eyes. Not only did Wolfe and Held find smaller tilt aftereffects under $\mathbf{M}-\mathbf{B}$ than under $\mathbf{M}-\mathbf{M}$ conditions, but alternate monocular adaptation without binocular adaptation yielded smaller aftereffects when the test grating was viewed by both eyes than when it was viewed by one eye. In contrast, Wade and Wenderoth (1978) obtained comparable tilt aftereffects under M-M, M-B, B-B, and B-M conditions. Although Moulden (1980) found smaller aftereffects for the M-B than for the M-M, B-B, or B-M 
combinations, he concluded that aftereffects were reduced under M-B conditions not because the test display was partly coded by purely binocular detectors that were not adapted during monocular stimulation, but because only one of the two monocular systems by which the test was coded was adapted.

Vidyasagar (1976) generated binocular-specific McCollough effects by means of a technique that seems open to more general use. In the standard McCollough effect, a vertical grating shown in white light appears blue and horizontal grating looks pink following alternate exposure to vertical lines in red light and horizontal lines in blue light. Vidyasagar ensured that each eye received similar exposure to red-vertical and blue-vertical stimulation, and to redhorizontal and blue-horizontal, by requiring observers to view a vertical grating in red light and a horizontal grating in blue light with each eye open in turn, but a vertical grating in blue light and a horizontal grating in red light with both eyes open. The color appearance of vertical and horizontal gratings shown in white light was then judged with one eye open and with both eyes open. Opposite monocular and binocular McCollough effects were obtained; for example, the vertical test grating appeared blue when viewed by either the left eye or the right eye but seemed pink when viewed with both eyes open.

The above aftereffects can be explained by supposing that different detector systems were adapted by inspection with one eye and with two eyes. Vidyasagar supposed that the monocular McCollough effects were mediated by cells that were responsive to both eyes but excited through one eye and inhibited through the other, whereas the binocular aftereffects reflected cells that exhibited synergistic facilitation in response when both eyes were simultaneously stimulated. These latter cells are unique binocular detectors in the sense described by Wolfe and Held (1981).

Recently, Anstis and Duncan (1983) reported separate motion aftereffects from each eye and from both eyes. Subjects viewed clockwise rotation of a patterned disk with the left eye, alternating with anticlockwise rotation viewed with the right eye. The resulting aftereffect was an anticlockwise motion aftereffect with the left eye, and a clockwise motion aftereffect with the right eye. In a second experiment, subjects adapted alternately to the following exposures: clockwise rotation left eye, clockwise right eye, and anticlockwise both eyes together. They saw an anticlockwise motion aftereffect with the left eye only or with the right eye only, and a clockwise motion aftereffect when both eyes were open. A model of monocular and binocular inputs to motion-sensitive neural channels was proposed.

Two experiments were undertaken using complementary monocular and binocular adaptation to study the McCollough effect and the motion after- effect. The aim of the first experiment was not only to replicate the phenomenon reported by Vidyasagar (1976), but to measure the orientation-selectivity and relative strength of the McCollough effects obtained with monocular and binocular exposure to the test stimulus. In the second experiment, observers adapted to a contracting (or expanding) spiral with each eye in turn and to an expanding (or contracting) spiral with both eyes open. The question asked was: would opposite directional aftereffects be found when the stationary test spiral was viewed monocularly and binocularly.

\section{EXPERIMENT 1}

\section{Method}

The six observers had normal color vision, spatial acuity of $20 / 20$ or better in each eye, and stereoacuity exceeding 40 secarc in disparity. Since the observers had participated in earlier McCollough effect experiments, they were experienced in providing magnitude estimates of the strength of the aftereffect.

Square-wave gratings of 1.2 cycles/deg spatial frequency and .8 contrast were projected onto a screen in a darkened room to subtend an angle of $20 \mathrm{deg} 42 \mathrm{~min} \times 14 \mathrm{deg} 30 \mathrm{~min}$ at a distance of $270 \mathrm{~cm}$. The color of the gratings was varied using Kodak Wratten gelatin filters with the following dominant wavelengths and CIE chromaticity coordinates under tungsten illumination: red (No. 26), $620.6 \mathrm{~nm}, X=.6815, Y=.3126$; green (No. 61), $533.8 \mathrm{~nm}, X=.2317, Y=.6910$. The projector had a 250-W tungsten illuminant. The observers, who were tested individually, were required to maintain an upright head and body position during both the adaptation and the test stages of the experiment.

The stimulus conditions changed every 30 sec during the 30-min adaptation period. For three observers, the stimulus sequence was: vertical grating in red light viewed with the left eye open, redvertical with right eye open, green-vertical with both eyes open, green-horizontal with left eye open, green-horizontal with right eye open, and red-horizontal with both eyes open. The reverse colortilt combinations were used for monocular and binocular viewing for the other three observers. Under monocular conditions, the nonviewing eye was completely occluded.

Test trials commenced $1 \mathrm{~min}$ after adaptation ceased. The observer judged the color appearance of laterally juxtaposed gratings, each subtending $10 \mathrm{deg} 21 \mathrm{~min} \times 14 \mathrm{deg} 30 \mathrm{~min}$, in white light. In addition to reporting whether each grating appeared green or pink/red, the observer estimated saturation on a scale ranging from 0 to 5 . Four test pairs (gratings tilted 0 vs. $90 \mathrm{deg}, 15 \mathrm{vs.}$ $105 \mathrm{deg}, 30$ vs. $120 \mathrm{deg}$, and 45 vs. $135 \mathrm{deg}$ clockwise from vertical) were used in order to establish the orientation selectivity of the color aftereffects. Test judgments were made in turn with the left eye open, the right eye open, and both eyes open. Four measures were obtained for each test stimulus under each viewing condition.

\section{Results}

Monocular and binocular adaptation to complementary color-tilt combinations yielded orientationselective color aftereffects for each observer in the manner that Vidyasagar (1976) has described. Thus observers exposed monocularly to red-vertical and binocularly to green-vertical reported that vertical lines shown in white light looked green when viewed with one eye open but pink when viewed with both eyes open. Since similar magnitude estimates were 


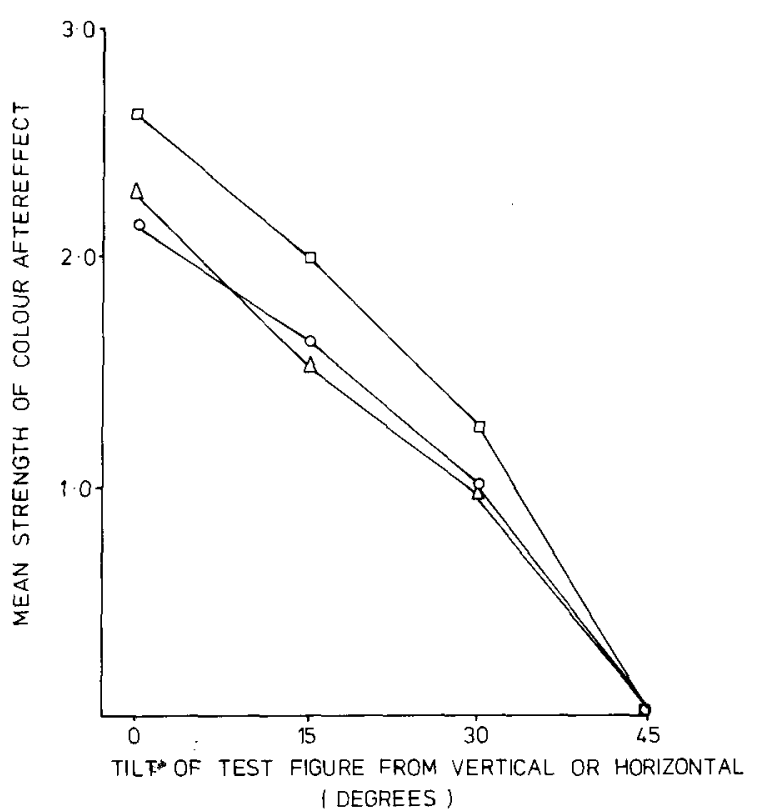

Figure 1. Mean estimated strength of the McCollough effect when test patterns at different orientations were viewed with the left eye open $(O-O)$, the right eye open $(\Delta-\Delta)$, and both eyes open $(\square-\square)$.

given for green and pink/red aftereffects, the two sets of results were pooled in data analysis. Figure 1 shows the mean estimate of the strength of aftereffects as a function of the viewing conditions and the orientation of the test gratings. Analysis of variance based on the 0-, 15-, and 30-deg measures showed that the McCollough effect dropped significantly in strength as the test gratings differed in orientation from the inspection gratings $[F(2,10)=$ $24.83, \mathrm{p}<.01$ ]. The means did not differ significantly between viewing conditions $[F(2,10)=2.22$, $p>.05]$, and the interaction between orientation difference and viewing conditions was not significant $[F(4,20)=0.41, p>.05]$. The monocular-contingent and the binocular-contingent McCollough effects thus showed similar orientation-selectivity. The drop to half strength over an orientation range of about $30 \mathrm{deg}$ matches the estimate of orientation tuning reported for the McCollough effect by Ellis (1977).

\section{EXPERIMENT 2}

Experiment 2 examined the possibility of inducing monocular-specific as well as binocular-specific motion aftereffects by successive monocular and binocular adaptation to lines moving in different directions, using conditions of stimulation analogous to those employed in Experiment 1.

\section{Method}

Six observers with acuity of $20 / 20$ or better in each eye and with normal stereopsis were tested, each over six sessions separated by
1 day or more. During adaptation, the observers viewed a threeturn spiral that was rotated either clockwise (so that it appeared to expand) or counterclockwise (contraction) at $85 \mathrm{rpm}$. The spiral, which was drawn in black ink on white paper with a contrast of 0.8 defined by (Imax - Imin)/(Imax + Imin), subtended 11 deg 21 min at a viewing distance of $150 \mathrm{~cm}$. The space-average luminance of the display was $13 \mathrm{~cd} / \mathrm{m}^{2}$. Following the 30 -min adaptation period, the observer made successive test judgments by looking at the stationary spiral for $5 \mathrm{sec}$ at a time with the left eye open, the right eye open, and both eyes open. In each case, the observer just reported whether the spiral appeared to be expanding or contracting, and then estimated the strength of the aftereffect on a scale ranging from 0 to 5 . Test judgments began 1 min following the end of the adaptation session. Eight judgments each were made with the left eye, the right eye, and both eyes, with $1 \mathrm{~min}$ between successive judgments under the same condition. Decay in aftereffects could thus be studied over $8 \mathrm{~min}$.

Three different adaptation conditions were employed. When opposite monocular and binocular stimulation was provided during adaptation, the observer viewed in sequence an expanding (or contracting) spiral with the left eye open, the same spiral with the right eye open, and a contracting (or expanding) spiral with both eyes open. The conditions of stimulation changed each $30 \mathrm{sec}$ throughout the 30-min adaptation period. In the two sessions given to each observer under this adaptation condition, one provided monocular-contraction and binocular-expansion stimulation and the other, monocular-expansion and binocular-contraction stimulation.

A monocular-alone adaptation condition was also employed, with the observer viewing a rotating spiral (contracting in one session and expanding in the other) for $30 \mathrm{sec}$ with the left eye open, then viewing the same stimulus for $30 \mathrm{sec}$ with the right eye open, and subsequently closing both eyes for $30 \mathrm{sec}$. This sequence was repeated throughout the 30 -min adaptation period. Under binocular-alone adaptation conditions, the observer spent the 30-min session in turn adapting for $30 \mathrm{sec}$ to a rotating spiral (contracting in one session and expanding in the other) and then sitting with both eyes closed for $60 \mathrm{sec}$. These different conditions were employed both to demonstrate that intermittent stimulation is sufficient to produce monocular and binocular aftereffects and to compare the size of the aftereffects found when the test figure is viewed with one eye and with two eyes after monocular and binocular adaptation.

\section{Results}

Figure 2 shows the mean strength of the aftereffect induced by exposure to apparently expanding and apparently contracting spirals under the three adaptation conditions (monocular-alone, binocular-alone, and complementary monocular and binocular stimulation). Measures are given separately for the test judgments that were made with the left eye open, the right eye open, and both eyes open at different times (1-2, 3-4, 5-6, and 7-8 $\mathrm{min})$ over the test phase. The direction of the aftereffect (expansion or contraction) is not shown in Figure 2 because in each case the mean aftereffect was one of expansion following exposure to an apparently contracting spiral and of contraction following exposure to an apparently expanding spiral. Adaptation to complementary monocular and binocular stimulation caused the stationary test spiral to seem to be, depending on the combination employed during adaptation, expanding when viewed monocularly and contracting when seen binocularly or contracting when viewed monocularly and expanding when seen 


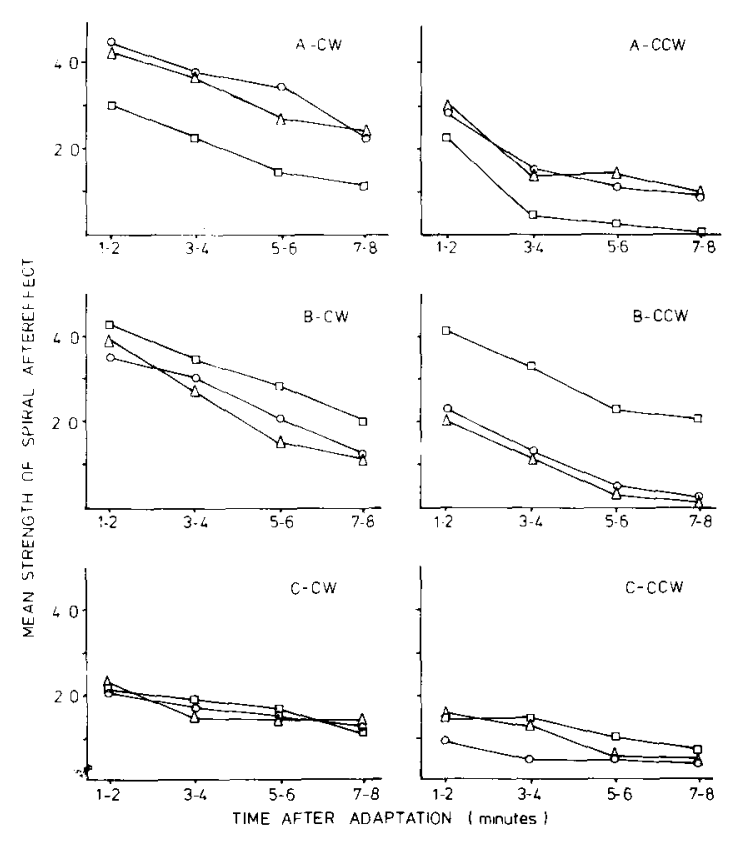

Figure 2. Mean estimated strength of the spiral aftereffect in the $8 \mathrm{~min}$ after adaptation. Judgments were made with the left eye open $(O-O)$, the right eye open $(\Delta-\Delta)$, and both eyes open ( $\square-\square)$ following monocular adaptation (A), binocular adaptation (B), and complementary monocular and binocular adaptation (C). Measures are shown separately for aftereffects induced by exposure to clockwise $(\mathrm{CW})$ and counterclockwise $(\mathrm{CCW})$ rotation of the spiral.

binocularly. The counterpart of the phenomenon demonstrated by Vidyasagar (1976) for the McCollough effect can thus be induced with the spiral aftereffect.

Since the direction of the aftereffect was consistently opposite to the direction of stimulation, the further comparisons were directed towards identification of variables that influenced the magnitude of the aftereffect. Analysis of variance involving repeated measures on all three independent variables was undertaken. Separate analyses were carried out for the aftereffect measures consequent upon monocular adaptation (Figure 2A), binocular adaptation (Figure 2B), and complementary adaptation (Figure $2 C$ ). In each case, the independent variables were direction of rotation of the spiral during adaptation (clockwise, counterclockwise), manner in which the test stimulus was viewed (left eye alone, right eye alone, both eyes), and delay in test judgments following adaptation (1-2, 3-4, 5-6, 7-8 $\mathrm{min})$.

Adaptation involving monocular stimulation without binocular stimulation yielded significantly larger aftereffects when the stationary test spiral was viewed by the left eye alone or the right eye alone than when viewed by both eyes together $[F(2,10)=$ $15.84, p<.01]$. In contrast, adaptation involving binocular stimulation without monocular stimulation produced larger aftereffects when the test grating was viewed by the two eyes together than when viewed by the left eye alone or the right eye alone $[F(2,10)=$ $5.06, \mathrm{p}<.05]$. Complementary adaptation resulted in aftereffects of comparable size when test judgments were made by either a single eye or both eyes $[F(2,10)$ $=0.20, p>.05]$. Adaptation to expansion yielded larger aftereffects than did adaptation to contraction in the case of monocular stimulation $[F(1,5)=12.13$, $\mathrm{p}<.05]$ and binocular stimulation $[\mathrm{F}(1,5)=8.04$, $\mathrm{p}<.05]$, but not complementary stimulation $[\mathrm{F}(1,5)$ $=3.77, p>.05]$. The magnitude of the aftereffect depended upon when, after adaptation, the test measures were obtained. Significant decay occurred following monocular stimulation $[F(3,15)=51.75$, $\mathrm{p}<.01]$, binocular stimulation $[\mathrm{F}(3,15)=99.09$, $\mathrm{p}<.01]$, and complementary stimulation $[\mathrm{F}(3,15)=$ $26.30, \mathrm{p}<.01]$. There was a significant interaction between direction of rotation during adaptation and manner of viewing the test spiral under conditions of monocular stimulation $[\mathrm{F}(2,10)=13.62, \mathrm{p}<.01]$ and binocular stimulation $[\mathrm{F}(2,10)=6.00, \mathrm{p}<.05]$. No other interaction was significant.

\section{DISCUSSION}

As first demonstrated by Vidyasagar (1976), the color-tilt relationship (McCollough effect) found following complementary monocular and binocular exposure to colored gratings depends on whether test stimuli are viewed by a single eye or by both eyes together. Monocular and binocular exposure to opposite directions of motion also yields aftereffects that differ in their directional properties in accordance with whether one eye or both eyes are used in viewing the stationary test display. Neither effect can be explained by assuming that binocular processing simply involves an additive combination of inputs from the two monocular channels. In the case of the motion aftereffect, the stimulation during the induction session was such that each monocular channel received the same amount of exposure to expansion and contraction. If nothing more than an additive combination of monocular inputs had occurred, either no aftereffect would have resulted (because the opposite effects canceled) or the same directional aftereffect would have been found irrespective of whether the test stimulus was viewed by a single eye or by both eyes together (in the event that one direction of motion generates stronger aftereffects than the other).

Opposite monocular and binocular aftereffects would be found following complementary stimulation if there were adaptation not only of detectors that are maximally excited by input to a single eye (either monocular-specific cells or binocular cells that are excited through one eye but inhibited through the other eye), but also binocular detectors that exhibited 
synergistic facilitation in response to simultaneous stimulation of both eyes. Complementary stimulation would produce selective directional adaptation within these two sets of detectors. For example, exposing each eye in turn to contraction but both eyes together to expansion would result in greater adaptation of monocular-contraction detectors than of monocular-expansion detectors, and greater adaptation of binocular-expansion detectors than of binocular-contraction detectors. If the nature of the aftereffect depends upon whether there is selective adaptation within the detector systems by which the test stimulus is normally signaled, the direction of distortion will vary in accordance with whether one eye or both eyes are used in viewing the test stimulus. Or, as proposed by the Anstis and Duncan (1983) model, there must be nonlinear, synergistic binocular units, which give only a weak response to stimulation from either eye alone, but a very much stronger response to simultaneous stimulation from the two eyes. The action threshold of such a unit is exceeded by binocular, but not monocular, inputs.

This line of argument can be applied to the differences in magnitude of aftereffect found when the test stimulus was viewed by one eye or by both eyes following monocular without binocular stimulation or binocular without monocular stimulation. The difference under such conditions was in the size rather than the direction of the aftereffect. Consistent with results obtained by Moulden (1980) and Wolfe and Held (1981) for the tilt aftereffect, the monocularbinocular combination yielded a smaller motion aftereffect than the monocular-monocular combination. This difference would be expected if there was less selective adaptation among the detectors coding the binocularly viewed test stimulus than among those coding the monocularly viewed test stimulus. In contrast to the result obtained by Moulden (1980) with the tilt aftereffect, we obtained a smaller motion aftereffect for the binocular-monocular combination than for the binocular-binocular combination. This difference might occur if there was some degree of uniqueness in monocular as well as binocular processing. The test stimulus viewed by one eye would thus be signaled by proportionately fewer adapted detectors than would the test stimulus viewed by both eyes.

The motion aftereffect diminished in strength over the test phase, but under most conditions an aftereffect was still evident 8 min after adaptation had ceased. Favreau (1979) and Masland (1969) found that the spiral aftereffect will persist for $24 \mathrm{~h}$ or more following continuous adaptation for $15 \mathrm{~min}$ to a rotating spiral. Although the adaptation period lasted $30 \mathrm{~min}$ in the present study, the stimulation was discontinuous. Monocular and binocular stimulation produced larger initial aftereffects than were found following complementary stimulation. Using monoc- ular adaptation, Bonnet and Pouthas (1972) demonstrated two phases in decay of the motion aftereffect. Fast, vivid movement was visible even on a noise background immediately following inspection of moving lines, but thereafter the aftereffect took the form of slower, less vivid movement that was seen best when the inspection and test stimuli were similar spatially. It is possible that motion aftereffects consequent upon complementary stimulation lack an initial nonselective stage. In addition, exposure to an apparently expanding spiral and to an apparently contracting spiral during complementary stimulation induced aftereffects of comparable size, while expansion produced the larger aftereffect in the case of monocular and binocular stimulation. This latter trend matches findings from other studies (e.g., Bakan \& Mizusawa, 1963; Scott, Lavender, McWhirt, \& Powell, 1966). There is no obvious reason why directional differences in magnitude were not obtained under the conditions of complementary stimulation.

The claim that complementary stimulation isolates unique monocular and binocular aspects of visual processing can be tested directly by means of comparisons between stereoblind and stereonormal observers. Stereoblind observers lack binocular detectors, but they have normal monocular functioning (see Lema \& Blake, 1977). When a person who is stereoblind is tested, alternate monocular and binocular exposure to opposite stimulation should yield either no motion aftereffect or a slight aftereffect in one direction (if either expansion or contraction is the more potent adaptation stimulus) irrespective of whether test judgments are made using one eye or both eyes. Furthermore, for stereoblind observers the size of the aftereffect following monocular without binocular stimulation or binocular without monocular stimulation should be independent of whether one eye or both eyes are used to view the test stimulus.

\section{REFERENCES}

Anstis, S., \& Duncan, K. (1983). Separate motion aftereffects from each eye and from both eyes. Vision Research, 23, 161-169.

Bakan, P., \& Mizusawa, K. (1963). Effect of inspection time and direction of rotation on a generalized form of the spiral aftereffect. Journal of Experimental Psychology, 65, 583-586.

Barlow, H. B., \& Brindley, G. S. (1963). Inter-ocular transfer of movement after-effect during pressure blinding of the stimulated eye. Nature, $200,1347$.

Blake, R., \& Fox, R. (1972). Interocular transfer of adaptation to spatial frequency during retinal ischemia. Nature New Bio$\log y, 240,76-77$.

Bonnet, C., \& Pouthas, V. (1972). Interactions between spatial and kinetic dimensions in movement aftereffect. Perception \& Psychophysics, 12, 193-200.

ElLIS, S. R. (1977). Orientation selectivity of the McCollough effect: Analysis, by equivalent contrast transformation. Perception \& Psychophysics, 22, 539-544.

Favreau, O. E. (1979). Persistence of simple and contingent motion aftereffects. Perception \& Psychophysics, 26, 187-194. 
Julesz, B. (1971). Foundations of cyclopean perception. Chicago: University of Chicago Press.

Lema, S. A., \& BLAKE, R. (1977). Binocular summation in normal and stereoblind humans. Vision Research, 17, 691-695.

Long, G. M. (1979). The dichoptic viewing paradigm: Do the eyes have it? Psychological Bulletin, 86, 391-403.

Masland, R. H. (1969). Visual motion perception: Experimental modification. Science, 165, 819-821.

Mitchell, D. E., \& WARE, C. (1974). Interocular transfer of a visual after-effect in normal and stereoblind humans. Journal of Physiology, 236, 707-721.

Mounden, B. (1980). After-effects and the integration of patterns of neural activity within a channel. Philosophical Transactions of the Royal Society, London, Series B, 290, 39-55.

Movshon, J. A., Chambers, B. E. I., \& Blakemore, C. (1972).
Interocular transfer in normal humans and those who lack stereopsis. Perception, 1, 483-490.

Scott, T. R., Lavender, A. D., McWhirt, R. A., \& Powell, D. A. (1966). Directional asymmetry of motion aftereffect. Journal of Experimental Psychology, 71, 806-815.

VIDYAsagar, T. R. (1976). Orientation specific colour adaptation at a central site. Nature, 261, 39-40.

WADE, N. J., \& WENDERoth, P. W. (1978). The influence of colour and contour rivalry on the magnitude of the tilt aftereffect. Vision Research, 18, 827-835.

Wolfe, J. M., \& Held, R. (1981). A purely binocular mechanism in human vision. Vision Research, 21, 1755-1759.

(Manuscript received June 15, 1983;

revision accepted for publication November 21, 1983.) 